\title{
Discontinuation of low-dose acetylsalicylic acid therapy in UK primary care: incidence and predictors in patients with cardiovascular disease
}

This article was published in the following Dove Press journal:

Pragmatic and Observational Research

15 March 2012

Number of times this article has been viewed

\author{
Elisa Martín-Merino' \\ Saga Johansson ${ }^{2}$ \\ Héctor Bueno ${ }^{3}$ \\ Luis A García Rodríguez' \\ 'Spanish Centre for \\ Pharmacoepidemiologic Research \\ (CEIFE), Madrid, Spain; ${ }^{2}$ AstraZeneca \\ R\&D, Mölndal, Sweden; ${ }^{3}$ Department \\ of Cardiology, Hospital General \\ Universitario Gregorio Marañón, \\ Madrid, Spain
}

Background: Discontinuation of low-dose acetylsalicylic acid (ASA) leads to an increased risk of cardiovascular and cerebrovascular events in patients taking low-dose ASA for secondary cardiovascular prevention. However, little is known about the rate of discontinuation in everyday clinical practice.

Objectives: To assess the rate of low-dose ASA discontinuation in primary care, and identify factors that predict discontinuation.

Methods: The Health Improvement Network, a large UK primary care database, was used to identify patients aged 50-84 years who received at least two consecutive prescriptions for lowdose ASA for secondary cardiovascular or cerebrovascular prevention in 2000-2007 ( $n=35,639)$. Discontinuation was defined as a period of at least 90 days after completion of the last prescribed course of ASA during which no repeat prescription was issued.

Results: During the study, 11,729 patients (32.9\%) discontinued ASA therapy (mean follow-up 2.5 years). The discontinuation rate was lower in patients with ASA indicated for myocardial infarction than for other indications. The diagnosis of gastrointestinal disorders during the study (overall odds ratio: 1.74; 95\% confidence interval: 1.61-1.88) was associated with increased rates of ASA discontinuation, whereas co-prescription of a proton pump inhibitor from the start of ASA therapy was associated with a decreased rate of discontinuation (odds ratio: $0.80 ; 95 \%$ confidence interval: $0.75-0.86$ ). Co-prescription of several other cardioprotective medications was also associated with a reduced risk of discontinuation, as were increasing age, prior hospitalization and overall number of co-medications

Conclusion: Continuous co-prescription of a PPI with low-dose ASA may improve adherence and outcomes, particularly in patients at both cardiovascular and gastrointestinal risk.

Keywords: aspirin, primary health care, compliance

\section{Introduction}

Long-term use of low-dose acetylsalicylic acid (ASA) is recommended for all patients with cardiovascular or cerebrovascular disease and no contraindications, and there is clear evidence for its efficacy in improving outcomes. ${ }^{1,2}$ Discontinuation of low-dose ASA therapy leads to a rapid increase in the risk of cardiovascular and cerebrovascular events (within 7-10 days), ${ }^{3-5}$ and recent evidence suggests that interruption of therapy may trigger a "prothrombotic rebound phenomenon" leading to an increase in risk over and above that present before starting therapy. ${ }^{6}$ A $20 \%$ rate of interruption or discontinuation of ASA therapy prescribed for secondary prevention of cardiovascular or cerebrovascular disease has been reported in secondary care in some clinical trials. ${ }^{7,8}$ However, there is still a lack of information about ASA discontinuation rates in everyday clinical practice.
Correspondence: Elisa Martín-Merino Spanish Centre for Pharmacoepidemiological Research (CEIFE), Almirante 28-2, E-28004, Madrid, Spain

Tel +34915313404

Fax +34 9| 53। 287|

Email elisaceife@gmail.com 
Adverse events are responsible for a substantial proportion of ASA discontinuations, ${ }^{9}$ with gastrointestinal disorders and upper gastrointestinal bleeding being commonly associated with ASA use. ${ }^{10}$ An increased risk of bleeding also often leads to discontinuation of low-dose ASA prior to surgery, although due to the risk of cardiovascular and cerebrovascular events, continuation of ASA therapy is now recommended in the majority of cases. ${ }^{11}$ Evidence suggests that the co-prescription of a proton pump inhibitor (PPI) may reduce the gastrotoxicity of ASA. ${ }^{12-14}$

As part of a study program assessing the outcomes of lowdose ASA discontinuation, we have examined the rate of discontinuation of low-dose ASA therapy in UK primary care, and identified the main factors which predict ASA discontinuation. We hypothesized that the diagnosis of gastrointestinal disorders during ASA therapy would be a significant predictor of discontinuation and, therefore, that co-prescription of PPIs would reduce the likelihood of discontinuation.

\section{Material and methods}

\section{Study population}

All patients who received at least two consecutive prescriptions for low-dose ASA (75-300 mg/day) for secondary prevention of cardiovascular or cerebrovascular disease from January 1, 2000 to December 31, 2007 were identified in The Health Improvement Network (THIN), a UK-based, anonymized primary care database. For inclusion in the study, patients were required to be aged 50-84 years, to have been enrolled with their primary care physician for at least 2 years, and to have a computerized prescription history of at least 1 year prior to their first ASA prescription. Patients were excluded if they had a record of ASA use before the study period, a history of alcohol abuse or alcohol-related disease, or a recorded diagnosis of cancer. The age range of 50-84 years was chosen in order to identify a population of patients receiving low-dose ASA for secondary cardiovascular or cerebrovascular disease prevention, with complete data recording. Cardiovascular and cerebrovascular event rates in younger patients are low, ${ }^{15}$ and older patients may have incomplete data recording since some individuals may reside in care homes.

\section{Data source}

THIN is a computerized medical research database containing systematically recorded, anonymized data on over 3 million patients currently registered with participating UK primary care practices. Patients included in the database are representative of the entire UK population with respect to age, sex, and geographical region. ${ }^{16}$ Information contained in THIN includes patient demographics, details of consultations with primary care physicians, information about consultant referrals and hospitalizations, laboratory test results, diagnoses, and prescriptions. THIN has been used in recent studies of ASA safety, ${ }^{14,17}$ and its validity for pharmacoepidemiological research has been demonstrated. ${ }^{18}$ This study was approved by a Multicenter Research Ethics Committee (08/H0305/49).

\section{Data collection and analysis}

Patient records were analyzed from 1 day after the first prescription of ASA (start date) until the earliest of the following endpoints: discontinuation of ASA therapy (defined as a period of $\geq 90$ days after the last prescribed course of ASA had been completed during which no repeat prescription was issued), recorded diagnosis of an exclusion factor (cancer, alcohol abuse, or alcohol-related disease), reaching the age of 85 years, death, or the end of the study period. Demographic and other patient characteristics collected were age, sex, socioeconomic status (Townsend deprivation index, ${ }^{19}$ recorded in THIN in quintiles taken from the UK national census, and urban/town fringe/rural location, recorded according to distance from amenities), body mass index, smoking history, alcohol use, low-dose ASA indication (four mutually exclusive categories: myocardial infarction, unstable angina, chronic ischemic heart disease, or cerebrovascular disease), number of primary care physician visits in the year prior to the start date, consultant referral or hospitalization before the start date (yes or no), comorbidities diagnosed prior to and during the study period, and concomitant medications prescribed during the study period. When there were two competing low-dose ASA indications, myocardial infarction was always considered the primary indication when present. If no myocardial infarction was present, the most recent indication was considered the primary indication.

The associations between ASA discontinuation and baseline characteristics, comorbidities diagnosed during ASA prescription, and co-medications used during ASA prescription were assessed using a nested case-control analysis. "Cases" were defined as patients who discontinued ASA therapy during the study period and "controls" as those who did not discontinue ASA during the study period. The index date for analysis was defined as the date on which the last ASA prescription ended for cases (discontinuers) and the date on which the last ASA prescription ended prior to a randomly chosen date for controls. For co-medication, current use at the index date was defined as use on the index 
date or within the previous 30 days, and use was analyzed relative to having received no prescription for the respective drug in the year prior to the index date (non-use). Additionally, for PPIs and $\mathrm{H}_{2}$ receptor antagonists $\left(\mathrm{H}_{2} \mathrm{RAs}\right)$, use since the start of ASA therapy was defined as use both within 30 days of the first ASA prescription and on the index date (or within the previous 30 days). In these patients, use since the start of therapy was divided into new use at the start date (no prescription in the 6 months prior to the first ASA prescription) and ongoing use at the start date (one or more prescriptions in the 6 months prior to the first ASA prescription).

\section{Statistical methods}

Odds ratios (OR) and 95\% confidence intervals (CI) were calculated using multivariate logistic regression adjusted for age at index date, sex, body mass index at the start date, time between the start and index dates, smoking history at the start date, number of primary care physician visits from 15 days to 1 year prior to the start date, referral or hospitalization (yes or no) from 15 days to 1 year prior to the start date, ASA indication, and the number of co-medications taken in the 60 days prior to the index date. All statistical procedures were performed with Stata (v 11.0; StataCorp LP, College Station, TX).

\section{Sensitivity analysis}

In order to verify the results, the analysis was repeated using a definition of discontinuation of a period of at least 30 days after the end of an ASA prescription during which no repeat prescription was issued.

\section{Results}

\section{Patient demographics and rate of discontinuation}

In total, 35,639 patients aged between 50 and 84 years (mean age $68.1 \pm 8.9$ years, $56.0 \%$ men) were identified who had received two or more consecutive prescriptions for low-dose ASA for secondary prevention of cardiovascular or cerebrovascular events during the study period (Table 1). Patients were followed up for a mean of 2.5 years, resulting in data for 89,246 person-years. The most common indication for ASA prescription was ischemic heart disease $(42.1 \%$ of patients), followed by cerebrovascular disease $(32.5 \%)$, myocardial infarction (23.5\%), and unstable angina (1.9\%). Just over half (51.7\%) of all patients had hypertension, 21.6\% had hyperlipidemia, and $19.3 \%$ were current smokers. Overall, 11,729 patients (32.9\%) discontinued ASA therapy at least once during the study period, giving a rate of
Table I Patient characteristics, ASA indication, and reason for termination of follow-up

\begin{tabular}{ll}
\hline Characteristic & $\begin{array}{l}\text { Total } \\
(\mathbf{n}=\mathbf{3 5}, 639) \mathbf{n}(\%)\end{array}$ \\
\hline Age at start date (years) & $13,092(36.7)$ \\
$50-64$ & $12,606(35.4)$ \\
$65-74$ & $9941(27.9)$ \\
$75-84$ & \\
Sex & $19,962(56.0)$ \\
Male & $15,677(44.0)$ \\
Female & \\
ASA indication & $8372(23.5)$ \\
Myocardial infarction & $680(1.9)$ \\
Unstable angina & $15,013(42.1)$ \\
Chronic ischemic heart disease & $11,574(32.5)$ \\
Cerebrovascular disease & \\
Cardiovascular risk factors & $18,443(51.7)$ \\
Hypertension & \\
Hyperlipidemia & \\
Current smoker & $7714(21.6)$ \\
Former smoker & $6861(19.3)$ \\
Reasons for termination of follow-up & $11,834(33.2)$ \\
ASA discontinuation & \\
Met exclusion criteria & \\
Reached 85 years of age & $11,729(32.9)$ \\
Death & $1989(5.6)$ \\
End of study period & $1912(5.4)$ \\
\hline
\end{tabular}

Notes: ${ }^{2}$ Alcohol abuse, alcohol-related disease or cancer diagnosis; ${ }^{b}$ diagnosed prior to the start date.

Abbreviation: ASA, acetylsalicylic acid.

discontinuation of 13.1 per 100 person-years $(95 \% \mathrm{CI}$ : 12.9-13.4).

Figure 1 shows Kaplan-Meier curves illustrating ASA discontinuation in the overall study population (Figure 1A) and according to indication (Figure 1B). Overall, the mean time to ASA discontinuation was 13.3 months (range 0.491.4 months). The rate of discontinuation of ASA therapy was higher in the first year of therapy than in subsequent years (incidence of 26.7 per 100 person-years in the first year [95\% CI: 26.1-27.3] versus 6.8 per 100 person-years in all subsequent years [95\% CI: 6.6-7.0]). Overall, $65.1 \%$ of all discontinuations (7636 patients) occurred in the first year of treatment, with a further $16.6 \%$ of discontinuations (1951 patients) occurring in the second year.

\section{Predictors of ASA discontinuation}

The likelihood of discontinuation by the end of the study was higher in younger patients and in women, relative to older patients and men, respectively (Table 2), but did not differ significantly according to socioeconomic level (data not shown). Hospitalization before the start date was associated with a lower likelihood of discontinuation than in patients who were not hospitalized before the start date. 


\section{A}

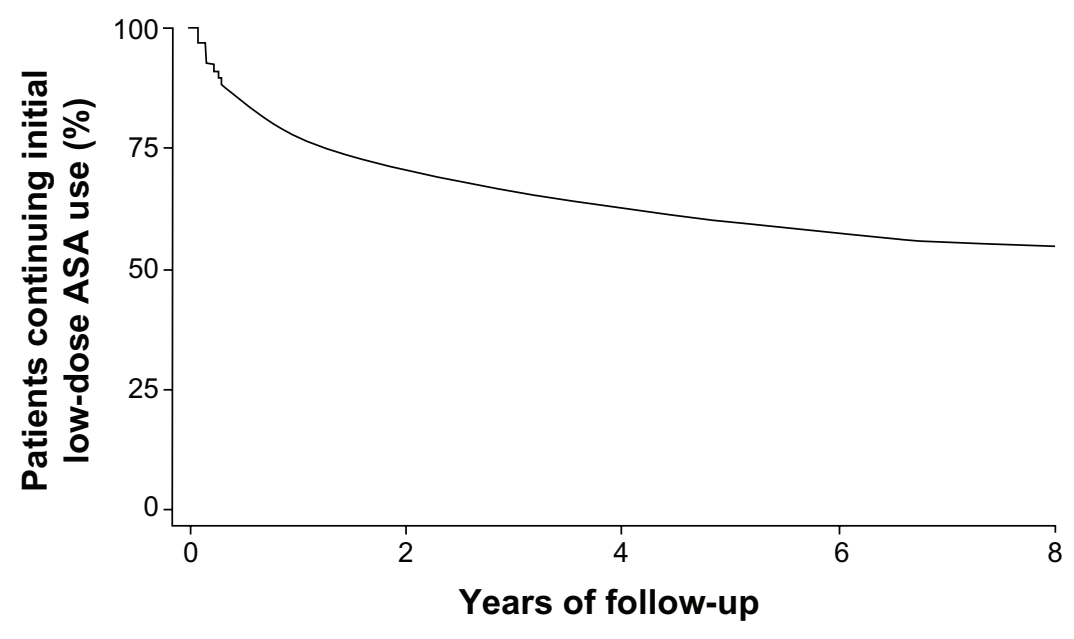

B

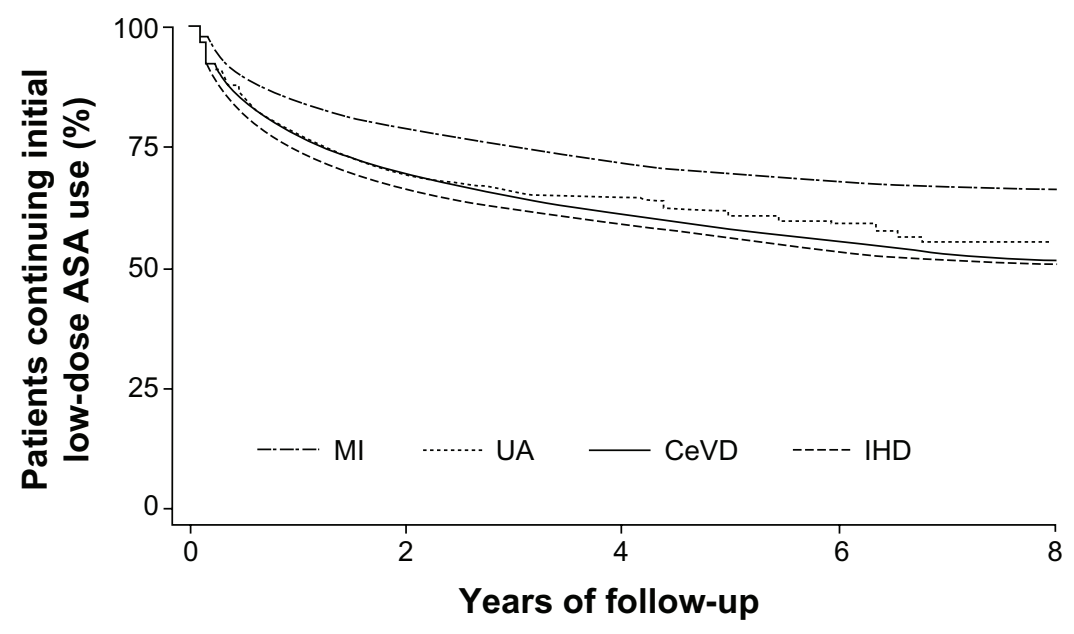

Figure I Kaplan-Meier survival curves illustrating acetylsalicylic acid discontinuation over the study period, in the whole cohort (A) and according to ASA indication (B). Abbreviations: ASA, acetylsalicylic acid; CeVD, cerebrovascular disease; IHD, ischemic heart disease; MI, myocardial infarction; UA, unstable angina.

Patients prescribed low-dose ASA after a myocardial infarction showed a significantly lower likelihood of discontinuation than those treated for other indications; overall, the proportion of patients who discontinued lowdose ASA therapy was $24.0 \%$ for those with myocardial infarction, $32.8 \%$ for those with cerebrovascular disease, $34.3 \%$ for those with unstable angina, and $37.9 \%$ for those with chronic ischemic heart disease.

Patients who developed upper gastrointestinal comorbidities or symptoms during the study had a significantly greater likelihood of ASA discontinuation than those who did not develop such comorbidities or symptoms (Table 3). Most notably, patients who received a diagnosis of peptic ulcer disease during ASA therapy were in excess of five times more likely to discontinue ASA treatment than those who did not (OR: 5.45, 95\% CI: 4.29-6.91). Associations between developing gastrointestinal comorbidities and discontinuing ASA therapy was also found in the subgroups of patients with different low-dose ASA indications (data not shown). The association between developing any gastrointestinal comorbidity and discontinuing ASA was OR: 1.57 (95\% CI: 1.33-1.85) for patients who had experienced a myocardial infarction, OR: 2.32 (95\% CI: 1.35-3.97) for patients with unstable angina, OR: 1.81 (95\% CI: 1.62-2.03) for patients with chronic ischemic heart disease, and OR 1.79 (95\% CI: 1.56-2.05) for patients with cerebrovascular disease. Other comorbidities were also associated with a significantly greater likelihood of ASA discontinuation, and it is notable that, with the exception of hypertension (OR: 0.91, 95\% CI: 0.85-0.97), no comorbidities (gastrointestinal or 
Table 2 Association between patient characteristics and ASA discontinuation risk

\begin{tabular}{|c|c|c|c|}
\hline & $\begin{array}{l}\text { ASA discontinuers } \\
(n=11,729) \\
n(\%)\end{array}$ & $\begin{array}{l}\text { ASA non-discontinuers } \\
(n=23,9 \mid 0) \\
n(\%)\end{array}$ & $\begin{array}{l}\text { Adjusted } O^{a} \\
(95 \% \mathrm{Cl})\end{array}$ \\
\hline \multicolumn{4}{|l|}{ Age at index date (years) } \\
\hline $50-64$ & $4288(36.6)$ & $6920(28.9)$ & $\mathrm{I} .00(-)$ \\
\hline $65-74$ & $3836(32.7)$ & $8498(35.5)$ & $0.79(0.75-0.84)$ \\
\hline $75-84$ & $3605(30.7)$ & $8492(35.5)$ & $0.73(0.69-0.78)$ \\
\hline \multicolumn{4}{|l|}{ Sex } \\
\hline Men & $6318(53.9)$ & $13,644(57.1)$ & $\mathrm{I} .00(-)$ \\
\hline Women & $54 I I(46.1)$ & $10,266(42.9)$ & $1.18(1.12-1.24)$ \\
\hline \multicolumn{4}{|l|}{ BMI $\left(\mathrm{kg} / \mathrm{m}^{2}\right)^{\mathrm{b}}$} \\
\hline $13-19$ & $304(2.6)$ & $637(2.7)$ & $0.90(0.78-1.05)$ \\
\hline $20-24$ & $288 I(24.6)$ & $5707(23.9)$ & $1.00(-)$ \\
\hline $25-29$ & $4165(35.5)$ & $8666(36.2)$ & $0.97(0.91-1.03)$ \\
\hline$\geq 30$ & $2479(21.1)$ & $5183(21.7)$ & $0.95(0.89-1.02)$ \\
\hline Unknown & $1900(16.2)$ & $3717(15.6)$ & $1.03(0.95-1.12)$ \\
\hline \multicolumn{4}{|l|}{ Smoking status ${ }^{\mathrm{b}}$} \\
\hline Non-smoker & $495 I(42.2)$ & $998 \mid(41.7)$ & $1.00(-)$ \\
\hline Current & $2448(20.9)$ & $4413(18.5)$ & $1.13(1.06-1.20)$ \\
\hline Former & $3648(31.1)$ & $8186(34.2)$ & $0.92(0.87-0.98)$ \\
\hline Unknown & $682(5.8)$ & $1330(5.6)$ & $1.12(1.00-1.25)$ \\
\hline \multicolumn{4}{|l|}{ Alcohol use (units/week) ${ }^{b}$} \\
\hline $0-1$ & $56 \mid 3(47.9)$ & I I,437 (47.8) & $1.00(-)$ \\
\hline $2-15$ & $3478(29.7)$ & $7045(29.5)$ & $0.99(0.94-1.05)$ \\
\hline $16-42$ & $983(8.4)$ & $1987(8.3)$ & $0.98(0.90-1.08)$ \\
\hline$\geq 43$ & $8(0.1)$ & $27(0.1)$ & $0.62(0.27-1.40)$ \\
\hline Unknown & $1647(14.0)$ & $34 \mid 4(14.3)$ & $0.92(0.84-1.00)$ \\
\hline \multicolumn{4}{|l|}{ Number of PCP visits ${ }^{c}$} \\
\hline $0-5$ & $3664(31.2)$ & $6951(29.1)$ & $1.00(-)$ \\
\hline $6-10$ & $3440(29.3)$ & $7019(29.4)$ & $0.95(0.89-1.09)$ \\
\hline$\geq 11$ & $4625(39.4)$ & $9940(41.6)$ & $0.94(0.89-1.00)$ \\
\hline \multicolumn{4}{|l|}{ Referral and hospitalization ${ }^{c}$} \\
\hline No & $9958(84.9)$ & I8,839 (78.8) & $1.00(-)$ \\
\hline Yes & $|77|(15.1)$ & $507 \mid(2 \mid .2)$ & $0.69(0.65-0.73)$ \\
\hline \multicolumn{4}{|c|}{ Number of concomitant medications ${ }^{d}$} \\
\hline $0-2$ & $4662(39.8)$ & $6443(27.0)$ & $1.00(-)$ \\
\hline $3-5$ & $3862(32.9)$ & $9605(40.2)$ & $0.6 I(0.57-0.64)$ \\
\hline $6-10$ & $2586(22.1)$ & $6494(27.2)$ & $0.64(0.60-0.68)$ \\
\hline$\geq 11$ & $619(5.3)$ & $1368(5.7)$ & $0.74(0.66-0.83)$ \\
\hline \multicolumn{4}{|l|}{ ASA indication } \\
\hline Myocardial infarction & $2007(17.1)$ & $6365(26.6)$ & $1.00(-)$ \\
\hline Unstable angina & $233(2.0)$ & 447 (1.9) & I.7I (1.44-2.03) \\
\hline Chronic IHD & $5687(48.5)$ & $9326(39.0)$ & $1.86(1.74-1.98)$ \\
\hline Cerebrovascular disease & $3802(32.4)$ & $7772(32.5)$ & $1.40(1.31-1.50)$ \\
\hline
\end{tabular}

Notes: aRelative to the indicated category (I.00 [-]). Adjusted for age at the index date, sex, BMI at the start date, time between the start and index dates, smoking history at the start date, number of PCP visits from 15 days to I year prior to the start date, referral or hospitalization (yes or no) from 15 days to I year prior to the start date, ASA indication and number of co-medications taken in the 60 days prior to the index date; ${ }^{b}$ at the start date; 'from I5 days to I year prior to the start date; ${ }^{\mathrm{d}}$ within 60 days prior to the index date.

Abbreviations: ASA, acetylsalicylic acid; $\mathrm{Cl}$, confidence interval; $\mathrm{BMI}$, body mass index; IHD, ischemic heart disease; OR, odds ratio; PCP, primary care physician.

otherwise) were associated with a lower likelihood of discontinuation.

Several drugs taken concomitantly with ASA at the index date (the date of ASA discontinuation, or a random date for controls) were associated with an increased likelihood of ASA discontinuation, including oral anticoagulants, antiinflammatory or anti-infective drugs, $\mathrm{H}_{2} \mathrm{RAs}$, hormone replacement therapy, and digoxin (Table 4). The greatest effect was observed in patients who received concomitant warfarin, which was associated with a seven-fold increase in the risk of ASA discontinuation (OR: $7.10,95 \% \mathrm{CI}$ : 6.26-8.05). The likelihood of ASA discontinuation was significantly lower in patients taking some other cardiovascular medications, including other antiplatelet drugs (most 
Table 3 Association between comorbidities ${ }^{\mathrm{a}}$ and ASA discontinuation risk

\begin{tabular}{|c|c|c|c|}
\hline & $\begin{array}{l}\text { ASA discontinuers } \\
(\mathrm{n}=11,729) \\
\mathrm{n}(\%)\end{array}$ & $\begin{array}{l}\text { ASA non-discontinuers } \\
(n=23,9 \mid 0) \\
n(\%)\end{array}$ & $\begin{array}{l}\text { Adjusted OR } \\
(95 \% \mathrm{Cl})\end{array}$ \\
\hline \multicolumn{4}{|c|}{ Upper gastrointestinal comorbidities or symptoms } \\
\hline Peptic ulcer disease & $202(1.7)$ & $115(0.5)$ & $5.45(4.29-6.91)$ \\
\hline Esophageal ulcer & $32(0.3)$ & $61(0.3)$ & $1.77(1.14-2.74)$ \\
\hline GERD & $415(3.5)$ & $959(4.0)$ & $1.30(1.15-1.47)$ \\
\hline Epigastric pain & $217(1.9)$ & $346(1.5)$ & $1.90(1.59-2.27)$ \\
\hline Dyspepsia/gastritis & $930(7.9)$ & $1730(7.2)$ & $1.69(1.54-1.84)$ \\
\hline Nausea & $247(2.1)$ & $515(2.2)$ & $1.43(1.22-1.68)$ \\
\hline Bloating & $128(I . I)$ & $286(1.2)$ & $1.40(1.12-1.73)$ \\
\hline Any of the above & $1482(12.6)$ & $2770(11.6)$ & $1.74(1.61-1.88)$ \\
\hline \multicolumn{4}{|l|}{ Other comorbidities } \\
\hline Hypertension & $2064(17.6)$ & $5889(24.6)$ & $0.91(0.85-0.97)$ \\
\hline Hyperlipidemia & $962(8.2)$ & $242 I(10.1)$ & I.05 (0.97-I.I5) \\
\hline Anemia & $328(2.8)$ & $54 I(2.3)$ & $1.91(1.65-2.20)$ \\
\hline Rheumatoid arthritis & $67(0.6)$ & $129(0.5)$ & $1.58(1.16-2.15)$ \\
\hline Heart failure & $313(2.7)$ & $679(2.8)$ & $1.47(1.27-1.69)$ \\
\hline Osteoarthritis & $777(6.6)$ & $1886(7.9)$ & $1.30(1.18-1.43)$ \\
\hline Asthma & $569(4.9)$ & $1418(5.9)$ & $1.21(1.09-1.35)$ \\
\hline COPD & $383(3.3)$ & 995 (4.2) & $1.13(1.00-1.29)$ \\
\hline Diabetes & II 43 (9.8) & $3038(12.7)$ & $1.02(0.94-1.10)$ \\
\hline
\end{tabular}

Notes: ${ }^{a}$ Diagnosed at any point during the study period; brelative to patients who never received a diagnosis for these conditions. Adjusted for age at the index date, sex, body mass index at the start date, time between the start and index dates, smoking history at the start date, number of primary care physician visits from 15 days to I year prior to the start date, referral or hospitalization (yes or no) from 15 days to I year prior to the start date, ASA indication and number of co-medications taken in the 60 days prior to the index date.

Abbreviations: ASA, acetylsalicylic acid; COPD, chronic obstructive pulmonary disease; Cl, confidence interval; GERD, gastroesophageal reflux disease; OR, odds ratio.

Table 4 Association between concomitant medications ${ }^{\mathrm{a}}$ and ASA discontinuation risk

\begin{tabular}{|c|c|c|c|}
\hline & $\begin{array}{l}\text { ASA discontinuers } \\
(n=I I, 729) \\
n(\%)\end{array}$ & $\begin{array}{l}\text { ASA non-discontinuers } \\
(n=23,9 \mid 0) \\
n(\%)\end{array}$ & $\begin{array}{l}\text { Adjusted } \mathrm{OR}^{\mathrm{b}} \\
(95 \% \mathrm{Cl})\end{array}$ \\
\hline \multicolumn{4}{|l|}{ Anti-inflammatory/analgesic drugs } \\
\hline Oral steroids & $436(3.7)$ & $752(3.2)$ & $1.43(1.25-1.62)$ \\
\hline NSAIDs (overall) & $1312(11.2)$ & $2335(9.8)$ & $1.27(1.18-1.37)$ \\
\hline Cyclo-oxygenase- 2 inhibitors & $285(2.4)$ & $347(1.5)$ & $1.80(1.53-2.13)$ \\
\hline Traditional NSAIDs & $1058(9.0)$ & $2026(8.5)$ & $1.15(1.06-1.25)$ \\
\hline Paracetamol & $2444(20.8)$ & $5716(23.9)$ & $1.03(0.96-1.09)$ \\
\hline \multicolumn{4}{|l|}{ Cardiovascular medications } \\
\hline Oral anticoagulants (warfarin) & 911 (7.8) & $394(1.7)$ & $7.10(6.26-8.05)$ \\
\hline Other antiplatelet drugs ${ }^{c}$ & $1293(11.0)$ & $3493(14.6)$ & $0.79(0.74-0.86)$ \\
\hline Antihypertensive drugs & $8690(74.1)$ & $20,509(85.8)$ & $0.63(0.59-0.67)$ \\
\hline Statins & $6543(55.8)$ & $|7,4| \mid(72.8)$ & $0.55(0.52-0.58)$ \\
\hline Nitrates & $2174(18.5)$ & $4919(20.6)$ & $0.88(0.83-0.94)$ \\
\hline Digoxin & $523(4.5)$ & $653(2.7)$ & $1.99(1.76-2.25)$ \\
\hline \multicolumn{4}{|l|}{ Gastroprotective medications } \\
\hline Proton pump inhibitors & $2385(20.3)$ & $5629(23.5)$ & $1.01(0.95-1.07)$ \\
\hline Histamine type 2 receptor antagonists & $469(4.0)$ & $802(3.4)$ & $1.23(1.09-1.39)$ \\
\hline Hormone replacement therapy ${ }^{d}$ & $288(5.3)$ & $310(3.0)$ & $1.51(1.27-1.80)$ \\
\hline Anti-infective drugs & $1406(12.0)$ & 3042 (12.7) & $1.14(1.05-1.22)$ \\
\hline Antidiabetic drugs & $1163(9.9)$ & 2735 (II.4) & $1.04(0.96-1.13)$ \\
\hline
\end{tabular}

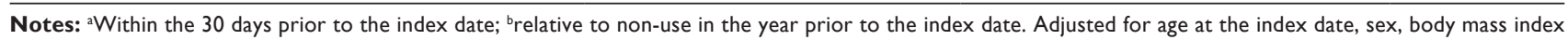
at the start date, time between the start and index dates, smoking history at the start date, number of primary care physician visits from 15 days to I year prior to the start date, referral or hospitalization (yes or no) from 15 days to 1 year prior to the start date, ASA indication, and number of co-medications taken in the 60 days prior to the index date. 'clopidogrel, dipyridamole, ticlopidine, cilostazol, anagrelide, abciximab, eptifibatide, tirofiban. The most common antiplatelet drug was clopidogrel ( $80.6 \%$ among discontinuers, $71.7 \%$ among non-discontinuers); ${ }^{d}$ percentages calculated from female patients only (ASA discontinuers, $\mathrm{n}=54 \mathrm{I}$; ; ASA nondiscontinuers, $n=10,266)$.

Abbreviations: ASA, acetylsalicylic acid; Cl, confidence interval; NSAIDs, non-steroidal anti-inflammatory drugs; OR, odds ratio. 
commonly clopidogrel), antihypertensive drugs, statins, and nitrates. Overall, no association was found between PPI use at the index date and ASA discontinuation.

Since gastroprotective medication may reduce the occurrence of gastrointestinal comorbidities, the association between the risk of ASA discontinuation and the use of PPIs and $\mathrm{H}_{2}$ RAs since the start of therapy (use at both the start date and the index date) was also analyzed (Table 5). The subgroup of patients who were receiving a PPI at the index date and who had been prescribed a PPI since the start of ASA therapy had a lower likelihood of ASA discontinuation than those who had not received a PPI in the year prior to the index date. In contrast, the use of $\mathrm{H}_{2} \mathrm{RAs}$ since the start of ASA therapy was not associated with a reduction in the rate of ASA discontinuation. The association between the use of PPIs since the start date with lower rates of ASA discontinuation was observed with both high and low-tomedium PPI doses (data not shown).

\section{Sensitivity analyses}

Reduction of the length of time without an ASA prescription which was considered "discontinuation" from 90 days to 30 days resulted in an increase in the total number of discontinuations to 18,872 (53.0\% of all patients). However, this had minimal effects on the predictors of ASA discontinuation. The factors significantly associated with ASA discontinuation remained largely the same (data not shown).

\section{Discussion}

In this study of over 35,000 patients aged 50-84 years who were prescribed low-dose ASA for secondary prevention of cardiovascular or cerebrovascular disease in 2000-2007, approximately one-third discontinued therapy for 90 days or more. This proportion increased to approximately $50 \%$ when the definition of discontinuation was changed to a period of 30 days or more without a new ASA prescription. Approximately two-thirds of all instances of ASA discontinuation occurred in the first year of therapy, and over $80 \%$ within the first 2 years. In agreement with our initial hypothesis, the diagnosis of peptic ulcer disease or the development of other upper gastrointestinal comorbidities or symptoms during ASA use were notably associated with an increased likelihood of ASA discontinuation, regardless of low-dose ASA indication.

The concomitant use of warfarin, digoxin, or anti-inflammatory drugs was also associated with a higher likelihood of ASA discontinuation. In contrast, the concomitant prescription of PPIs from the start of ASA therapy was associated with a decreased risk of ASA discontinuation, as were the concomitant use of some cardiovascular drugs, and an ASA indication of myocardial infarction. Other factors associated with an increased risk of ASA discontinuation included several non-gastrointestinal comorbidities, being female and being a current smoker, whereas older patients, former smokers, patients who had been admitted to hospital, patients with hypertension, and those receiving greater numbers of co-medications were all at a lower risk of discontinuation. The lower risk of low-dose ASA discontinuation seen in former smokers compared with current smokers may reflect a better understanding of cardiovascular risk in patients who succeed in giving up cigarettes.

In a separate analysis of the data in this study program assessing low-dose ASA discontinuation, ASA discontinuation has been shown to be associated with an approximately $40 \%$ increase in the risk of both myocardial infarction ${ }^{20}$ and stroke. ${ }^{21}$ Preventing discontinuation

Table 5 Association between use of gastroprotective medication since the start of ASA therapy and discontinuation risk

\begin{tabular}{|c|c|c|c|}
\hline & $\begin{array}{l}\text { ASA discontinuers } \\
(n=11,729) n(\%)\end{array}$ & $\begin{array}{l}\text { ASA non-discontinuers } \\
(n=23,9 \mid 0) n(\%)\end{array}$ & $\begin{array}{l}\text { Adjusted } \mathrm{OR}^{\mathrm{b}} \\
(95 \% \mathrm{Cl})\end{array}$ \\
\hline \multicolumn{4}{|c|}{ Proton pump inhibitors } \\
\hline Overall & $1585(13.5)$ & $4338(18.1)$ & $0.80(0.75-0.86)$ \\
\hline Ongoing use ${ }^{c}$ & $1246(10.6)$ & $3040(12.7)$ & $0.89(0.83-0.96)$ \\
\hline New use ${ }^{d}$ & $339(2.9)$ & $1298(5.4)$ & $0.59(0.52-0.67)$ \\
\hline \multicolumn{4}{|c|}{ Histamine type 2 receptor antagonists } \\
\hline Overall & $324(2.8)$ & $578(2.4)$ & $1.20(1.04-1.39)$ \\
\hline Ongoing use & $278(2.4)$ & $486(2.0)$ & $1.22(1.04-1.42)$ \\
\hline New use & $46(0.4)$ & $92(0.4)$ & $1.12(0.77-1.61)$ \\
\hline
\end{tabular}

Notes: ase within both the first and last 30 days of ASA therapy; brelative to non-use in the year prior to the index date. Adjusted for age at the index date, sex, body mass index at the start date, time between the start and index dates, smoking history at the start date, number of primary care physician visits from 15 days to $I$ year prior to the start date, referral or hospitalization (yes or no) from 15 days to I year prior to the start date, ASA indication, and number of co-medications taken in the 60 days prior to the index date; 'at least one proton pump inhibitor prescription within the 6 months prior to the first low-dose ASA prescription; ${ }^{d}$ no proton pump inhibitor prescription within the 6 months prior to the first low-dose ASA prescription.

Abbreviations: ASA, acetylsalicylic acid; $\mathrm{Cl}$, confidence interval; OR, odds ratio. 
should therefore be considered of great importance for improving outcomes in this patient population. To our knowledge, this is the first study to examine the rate of ASA discontinuation in primary care, and the rate of discontinuation we have found is higher than that reported in clinical trials (approximately 20\%). ${ }^{7,8}$ This probably reflects the contrast between well-controlled trial conditions with highly motivated patients and the situation in routine clinical practice.

The use of $\mathrm{H}_{2} \mathrm{RAs}$ at the index date or since the start of ASA therapy was not associated with a reduced rate of ASA discontinuation. $\mathrm{H}_{2}$ RAs are less effective than PPIs at reducing acid secretion, and current guidelines recommend the prescription of PPIs but not $\mathrm{H}_{2} \mathrm{RAs}$ to reduce ASA-related gastrointestinal injury. ${ }^{22,23}$ Adverse effects associated with PPI use are rare, ${ }^{24}$ and although some pharmacodynamics studies have suggested that PPIs reduce the efficacy of the antiplatelet drug clopidogrel if co-prescribed, the results of randomized and observational studies assessing this are conflicting, with considerable heterogeneity between studies. ${ }^{25}$ Patients with a low-dose ASA indication of myocardial infarction were less likely to discontinue therapy than those with other indications, which may be related to a greater perception of the severity and risk of recurrence of myocardial infarction, or a greater understanding of the benefits of ASA for the treatment of this condition. The concomitant prescription of warfarin was associated with a seven-fold increased rate of ASA discontinuation, which can largely be explained by the increased risk of bleeding. However, a lack of understanding of the rationale for co-prescribing an anticoagulant and antiplatelet agent may also have contributed to the increased rate of discontinuation. Conversely, the concomitant use of most cardiovascular therapies, such as other antiplatelet drugs or statins, was associated with a lower rate of ASA discontinuation. This may be because patients prescribed more than one anticoagulant therapy reflect a population with more severe disease who have a greater awareness of the risks associated with ASA discontinuation.

This study was performed in a large patient cohort representative of the whole of UK primary care, ${ }^{18}$ and over a long follow-up period. The validity of the results is supported by sensitivity analysis, which showed an increase in the absolute number of ASA discontinuations but did not change the associations with predictive factors. The accuracy of the results may have been limited by the fact that patients were assumed to adhere completely to their prescribed ASA therapy during the time covered by an ASA prescription, and never to use over-the-counter ASA. While patients aged over 65 years in the UK receive free prescriptions, and primary healthcare is easily accessed, some studies have reported that $20 \%-40 \%$ of patients who are receiving low-dose ASA do not receive it via prescription. ${ }^{26-28}$ Other medications, including some PPIs, are also available over the counter in the UK.

In conclusion, several factors influence the rate of ASA discontinuation, including the development of gastrointestinal comorbidities and the continuous co-prescription of a PPI since the start of therapy. In order to improve outcomes in this patient population, patient education about the risks associated with ASA discontinuation should be improved, and monitoring of continued ASA therapy should be increased. ${ }^{29}$ PPI co-prescription with ASA may also increase adherence, particularly in patients at both cardiovascular and gastrointestinal risk.

\section{Acknowledgments}

Elisa Martín-Merino contributed to the study design, data collection, statistical analysis, interpretation of data, and drafting the report; Luis A García Rodríguez contributed to data collection, statistical analysis, and reviewing the report; Saga Johansson and Héctor Bueno contributed to the study design, interpretation of data, and reviewing the report.

\section{Disclosure}

This work was supported by an unrestricted research grant from AstraZeneca R\&D, Mölndal. Luis A García Rodríguez and Elisa Martín-Merino work for the Spanish Centre for Pharmacoepidemiologic Research, which has received research funding from AstraZeneca R\&D, Mölndal. Luis A García Rodríguez has also received honoraria for serving on a scientific advisory board for AstraZeneca. Héctor Bueno has received honoraria for talks and advisory work from Almirall, Bayer, BMS, Daichii-Sankyo, Eli-Lilly, and SanofiAventis, and research grants from AstraZeneca. Saga Johansson is an employee of AstraZeneca R\&D, Mölndal. All authors had full access to all of the data (including statistical reports and tables) in the study and can take responsibility for the integrity of the data and the accuracy of the data analysis.

We acknowledge and thank Dr Stephen Sweet and Dr Catherine Hill of Oxford PharmaGenesis ${ }^{\mathrm{TM}}$ Ltd, who provided writing assistance also funded by AstraZeneca R\&D, Mölndal. 


\section{References}

1. Graham I, Atar D, Borch-Johnsen K, et al; for European Society of Cardiology (ESC); European Association for Cardiovascular Prevention and Rehabilitation (EACPR); Council on Cardiovascular Nursing; European Association for Study of Diabetes (EASD); International Diabetes Federation Europe (IDF-Europe); European Stroke Initiative (EUSI); International Society of Behavioural Medicine (ISBM); European Society of Hypertension (ESH); European Society of General Practice/Family Medicine (ESGP/FM/WONCA); European Heart Network $(\mathrm{EHN})$. European guidelines on cardiovascular disease prevention in clinical practice: executive summary. Eur Heart J. 2007;28(19):2375-2414.

2. Antithrombotic Trialists' (ATT) Collaboration. Aspirin in the primary and secondary prevention of vascular disease: collaborative metaanalysis of individual participant data from randomised trials. Lancet. 2009;373:1849-1860.

3. Maulaz AB, Bezerra DC, Michel P, Bogousslavsky J. Effect of discontinuing aspirin therapy on the risk of brain ischemic stroke. Arch Neurol. 2005;62(8):1217-1220.

4. Ferrari E, Benhamou M, Cerboni P, Marcel B. Coronary syndromes following aspirin withdrawal: a special risk for late stent thrombosis. J Am Coll Cardiol. 2005;45(3):456-459.

5. Biondi-Zoccai GG, Lotrionte M, Agostoni P, et al. A systematic review and meta-analysis on the hazards of discontinuing or not adhering to aspirin among 50,279 patients at risk for coronary artery disease. Eur Heart J. 2006;27(22):2667-2674.

6. Lordkipanidze M, Diodati JG, Pharand C. Possibility of a rebound phenomenon following antiplatelet therapy withdrawal: a look at the clinical and pharmacological evidence. Pharmacol Ther. 2009;123(2): 178-186.

7. De Schryver EL, van Gijn J, Kappelle LJ, Koudstaal PJ, Algra A; for Dutch TIA Trial and SPIRIT study groups. Non-adherence to aspirin or oral anticoagulants in secondary prevention after ischaemic stroke. J Neurol. 2005;252(11):1316-1321.

8. Newby LK, Bhapkar MV, White HD, et al; for SYMPHONY and 2nd SYMPHONY Investigators. Aspirin use post-acute coronary syndromes: intolerance, bleeding and discontinuation. J Thromb Thrombolysis. 2003;16(3):119-128.

9. Herlitz J, Toth PP, Naesdal J. Low-dose aspirin therapy for cardiovascular prevention: quantification and consequences of poor compliance or discontinuation. Am J Cardiovasc Drugs. 2010;10(2):125-141.

10. García Rodríguez LA, Hernández-Díaz S, de Abajo FJ. Association between aspirin and upper gastrointestinal complications: systematic review of epidemiologic studies. Br J Clin Pharmacol. 2001;52(5): 563-571.

11. Collet JP, Montalescot G, Blanchet B, et al. Impact of prior use or recent withdrawal of oral antiplatelet agents on acute coronary syndromes. Circulation. 2004;110(16):2361-2367.

12. Yeomans N, Lanas A, Labenz J, et al. Efficacy of esomeprazole (20 mg once daily) for reducing the risk of gastroduodenal ulcers associated with continuous use of low-dose aspirin. Am J Gastroenterol. 2008;103(10):2465-2473.

13. Scheiman JM, Devereaux PJ, Herlitz J, et al. Prevention of peptic ulcers with esomeprazole in patients at risk for ulcer development treated with low-dose acetylsalicylic acid: a randomised, controlled trial (OBERON). Heart. 2011;97(10):797-802.
14. Lin KJ, Hernandez-Diaz S, Rodriguez LA. Acid suppressants reduce risk of gastrointestinal bleeding in patients on antithrombotic or antiinflammatory therapy. Gastroenterology. 2011;141:71-79.

15. Hayden M, Pignone M, Phillips C, Mulrow C. Aspirin for the primary prevention of cardiovascular events: a summary of the evidence for the U.S. Preventive Services Task Force. Ann Intern Med. 2002;136(2): 161-172.

16. Bourke A, Dattani H, Robinson M. Feasibility study and methodology to create a quality-evaluated database of primary care data. Inform Prim Care. 2004;12(3):171-177.

17. García Rodríguez LA, Lin KJ, Hernández-Díaz S, Johansson S. Risk of upper gastrointestinal bleeding with low-dose acetylsalicylic acid alone and in combination with clopidogrel and other medications. Circulation. 2011;123:1108-1115.

18. Lewis JD, Schinnar R, Bilker WB, Wang X, Strom BL. Validation studies of the health improvement network (THIN) database for pharmacoepidemiology research. Pharmacoepidemiol Drug Saf. 2007;16(4): 393-401.

19. Morris R, Carstairs V. Which deprivation? A comparison of selected deprivation indexes. J Public Health Med. 1991;13(4):318-326.

20. García Rodríguez LA, Cea-Soriano L, Martin-Merino E, Johanssen S. Discontinuation of low dose aspirin and risk of myocardial infarction: case-control study in UK primary care. BMJ. 2011;343:d4094.

21. García Rodríguez LA, Cea Soriano L, Hill C, Johanssen S. Increased risk of stroke after discontinuation of acetylsalicylic acid: a UK primary care study. Neurology. 2011;76(8):740-746.

22. Lanza FL, Chan FK, Quigley EM; for Practice Parameters Committee of the American College of Gastroenterology. Guidelines for prevention of NSAID-related ulcer complications. Am J Gastroenterol. 2009;104(3):728-738.

23. Abraham NS, Hlatky MA, Antman EM, et al; for ACCF/ACG/AHA. ACCF/ACG/AHA 2010 Expert Consensus Document on the concomitant use of proton pump inhibitors and thienopyridines: a focused update of the ACCF/ACG/AHA 2008 expert consensus document on reducing the gastrointestinal risks of antiplatelet therapy and NSAID use: a report of the American College of Cardiology Foundation Task Force on Expert Consensus Documents. Circulation. 2010;122(24):2619-2633.

24. Yang YX, Metz DC. Safety of proton pump inhibitor exposure. Gastroenterology. 2010;139(4):1115-1127.

25. Kwok CS, Loke YK. Meta-analysis: effects of proton pump inhibitors on cardiovascular events and mortality in patients receiving clopidogrel. Aliment Pharmacol Ther. 2010;31(8):810-823.

26. Bedson J, Whitehurst T, Lewis M, Croft P. Factors affecting over-thecounter use of aspirin in the secondary prophylaxis of cardiovascular disease. Br J Gen Pract. 2001;51(473):1001-1003.

27. Campbell NC, Thain J, Deans HG, Ritchie LD, Rawles JM. Secondary prevention in coronary heart disease: baseline survey of provision in general practice. BMJ. 1998;316(7142):1430-1434.

28. Hopper S, Pierce M. Aspirin after myocardial infarction: the importance of over-the-counter use. Fam Pract. 1998;15 Suppl 1:S10-S13.

29. Haynes RB, Yao X, Degani A, Kripalani S, Garg A, McDonald HP. Interventions to enhance medication adherence. Cochrane Database Syst Rev. 2005;(4):CD000011.

Pragmatic and Observational Research

\section{Publish your work in this journal}

Pragmatic and Observational Research is an international, peer-reviewed, open access journal that publishes data from studies designed to reflect more closely medical interventions in real-world clinical practice compared with classical randomized controlled trials (RCTs). The manuscript management system is completely online and includes a very quick and fair peer-review

Submit your manuscript here: http://www.dovepress.com/pragmatic-and-observational-research-journal

system. Visit http://www.dovepress.com/testimonials.php to read real quotes from published authors. 